\title{
Optimization of green and deep green GaInN/GaN light emit- ting diodes for homogeneity
}

\author{
C. Wetzel ${ }^{* 1,2}$, P. Li ${ }^{1}$, T. Detchprohm ${ }^{1}$, and J. S. Nelson ${ }^{1}$ \\ ${ }^{1}$ Uniroyal Optoelectronics, 3401 Cragmont Dr, Tampa Florida 33619, USA \\ ${ }^{2}$ Future Chips Constellation, Rensselaer Polytechnic Institute, 110 8th Street, \\ Troy, New York 12180-3590, USA
}

Received 12 July 2004, revised 29 September 2004, accepted 22 October 2004

Published online 8 February 2005

PACS 78.55.Cr, 85.60.Jb

Development progress of high power $525 \mathrm{~nm}$ green LEDs employing GaInN/GaN active layers optimized for high morphological homogeneity is presented. We analyze the benefits of this process for the reproducibility in epitaxial growth and processing as well as across the wafer uniformity. We show that by means of our approach performance in terms of dominant wavelength and output power are highly reproducible across the wafer and from run to run. This lends special relevance also to any spectroscopic analysis of such sample material.

() 2005 WILEY-VCH Verlag GmbH \& Co. KGaA, Weinheim

\section{Introduction}

Progress of light emission efficiency in the green and deep green spectral range is crucial for the success of all solid state lighting. High performance GaInN/GaN quantum well (QW) based light emitting diodes (LEDs) have led to a wide range of commercial applications [1-4]. Yet, power performance in the green and deep green spectral range is still lagging far behind the development of red and blue. Among the key problems is the observation of a steep roll-off in emission power efficiency when the wavelength is increased from $460 \mathrm{~nm}$ in the blue to $525 \mathrm{~nm}$ in the green and $545 \mathrm{~nm}$ in the deep green. The physical reason behind this behavior needs to be identified to overcome such limitations. Many studies in this direction have been hampered by a limited set of available sample data. A meaningful study requires a large base of sample data to meliorate the effects of sample variations from growth run to growth run. In a large scale optimization process for green LED dies we have explored the benefits of different optimization priorities. In a first paradigm we have expanded the successful recipes for $395 \mathrm{~nm}$ and $470 \mathrm{~nm}$ blue LEDs towards the green by purposeful extension of natural process fluctuations resulting in longer wavelength photoluminescence. Among the key parameters we identified the growth temperature of the GaInN wells, well width, and to a lesser extend the TMIn flow rate during growth of the wells. Optimization towards intense narrow bandwidth green PL emission of bare MQW samples, however proofed misleading in the quest for green LEDs once these structure were imbedded in full pn-junctions for electric carrier injection. It was not until a rigorous optimization towards the smoothest growth morphology in the active region that $525 \mathrm{~nm}$ LED emission at an (un-encapsulated) bare die emission rate of $2 \mathrm{~mW}$ and beyond was achieved. These results have been summarized in a recent publication [5]. Here we provide details of the optimization progress leading to high yield production scale device manufacture.

"Corresponding author: e-mail: Wetzel@ieee.org, Phone: +1 518276 3755, Fax: +1 5182768042 

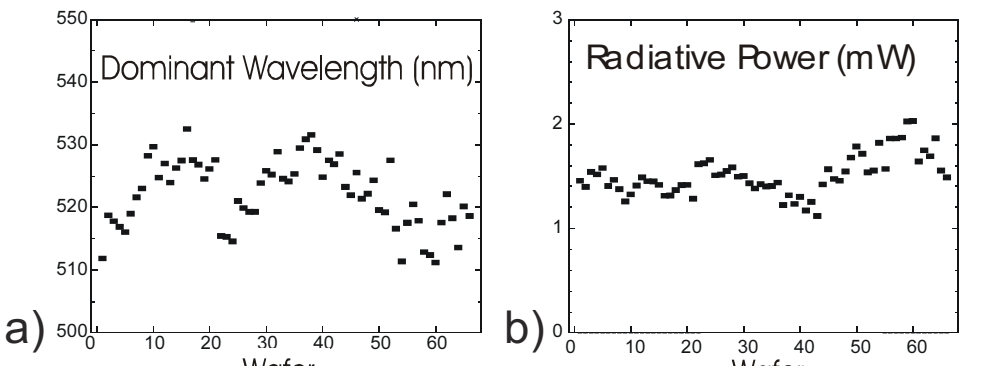

Fig. 1 Run to run evolution of electrooptical LED die wafer parameters given 846point medians across 2-inch wafers: dominant wavelength (a), radiative power (b), forward voltage at 20
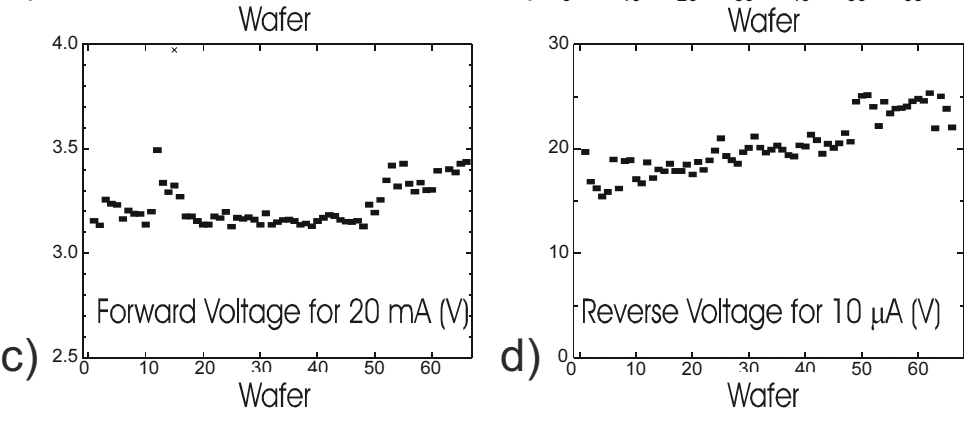
$\mathrm{mA}$ (c), reverse voltage at $10 \mu \mathrm{A}(\mathrm{d})$.

\section{Experimental}

The epitaxial growth of highly lattice mismatched materials has proven notoriously difficult throughout the literature. The large lattice mismatch and in addition very different thermo-dynamical properties of $\mathrm{GaN}$ and InN make epitaxial growth of their ternary alloys a delicate task. The technological relevant benefit of a strong optical bandgap variation with alloy composition complicate the development of stable reproducible emission wavelength devices due to these very variations. The technological advantage transposes into a production challenge.

For this study full LED epi wafers have been grown by metal organic vapor phase epitaxy in an Emcore D-180 Spectra GaN rotating disc multiwafer system using trimethyl and diethyl adducts of $\mathrm{Ga}$, In, $\mathrm{Al}$, as well as ammonia. $\mathrm{Ga}_{1-x} \mathrm{In}_{x} \mathrm{~N} / \mathrm{GaN}$ multiple $\mathrm{QW}$ (MQW) structures have been embedded in pndiodes on $<0001>$ sapphire substrate. Typical design parameters for the active region are as follows. Five

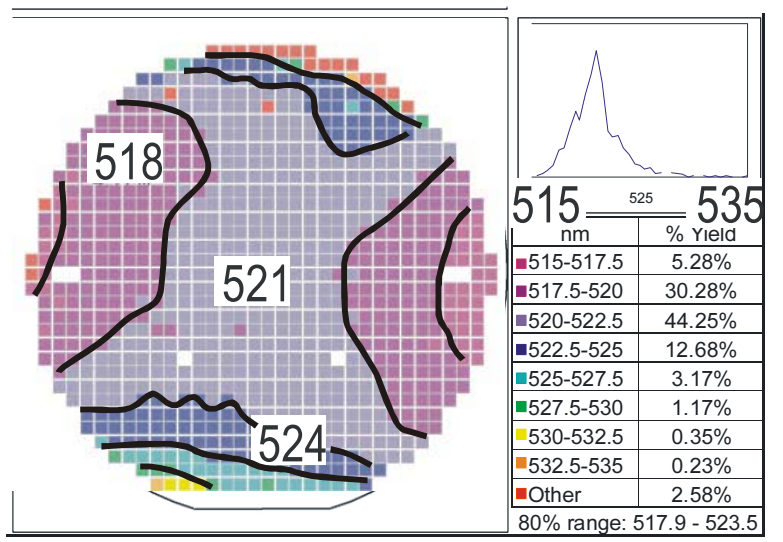

Fig. 2 Full wafer map of LED die performance of dominant wavelength $(\mathrm{nm})$. The inset shows the die distribution thereof.
$\mathrm{Ga}_{1-x} \mathrm{In}_{\mathrm{x}} \mathrm{N} / \mathrm{GaN} \mathrm{QW}$ s of nominal well width $3 \mathrm{~nm}$, separated by barriers of nominal $11 \mathrm{~nm}$ have been grown at temperatures of $650-850{ }^{\circ} \mathrm{C}$. Samples have been characterized at various stages of processing. The date presented here stems from fully processed but unseparated bare dies without coating.

Typical die relevant characterization parameters are summarized in Fig. 1 as a function of produced wafer number in a long period of consecutive epitaxial growth runs. We give the 846-point full wafer medians of the simultaneously measured quantities for a drive current of $20 \mathrm{~mA}$ : the dominant wavelength (a), the radiative power emitted within the 


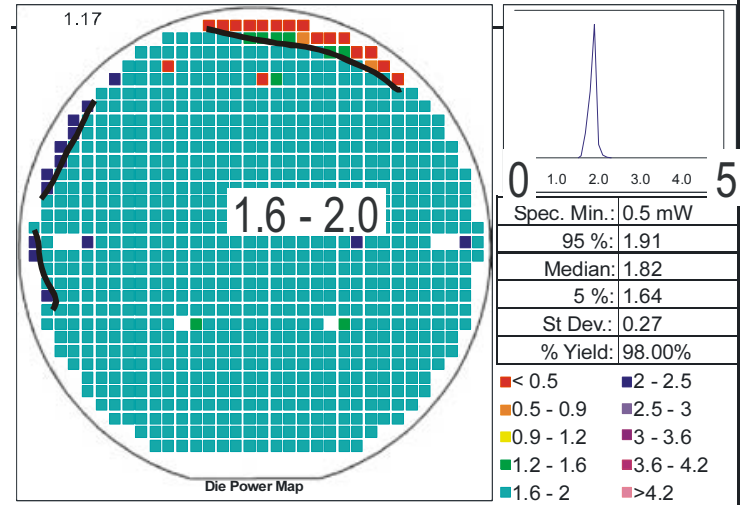

Fig. 3 Full wafer map of LED die performance of emitted power in the green spectral range $(\mathrm{mW})$ at $20 \mathrm{~mA}$. The inset shows the die distribution thereof. until reproducibility and wafer scale homogeneity prove a process production viable. In our case, key to high performance was the morphological optimization to the very low roughness of the growth surface to values as low as $0.14 \mathrm{~nm}$ root mean square (RMS). These values were assessed by atomic force microscopy (AFM) over an area of $5 \times 5 \mathrm{~m}^{2}$ after the last barrier of the MQW. This was primarily achieved by means of an optimization of the growth conditions using the typical parameters of gas flow, pressure and temperature. The goal was to avoid a decoration of threading dislocations with V-shaped defects [6] during the growth of QWs and barriers. The density of threading defects was unaffected. This improvement resulted in a decrease of the observable V-defect density. A detailed discussion of this aspect can be found in Ref. [5].

Full wafer maps of the dominant wavelength across the 2-inch wafer is presented in Fig. 2. Bins of the dominant wavelength are $3 \mathrm{~nm}$ wide. Colored areas of adjacent bins are delineated in black for better clarity. The inset gives the distribution of dies along the dominant wavelength. We find that $80 \%$ of the dies are within the very narrow spectral range from 517.9-523.5 nm. Visible emitted power of the identical processed wafer is given in Fig. 3. Here we find a consistent high power emission all across the wafer within the bin of 1.6-2.0 mW (5\%-tile $1.64 \mathrm{~mW}, 95 \%$-tile $1.91 \mathrm{~mW}$ with a median of $1.82 \mathrm{~mW}$ ).

Beyond relevance for commercial exploitation these results lend relevance also to the scientific exploitation of sample material obtained under these conditions.

\section{Summary}

In summary we have presented results on the progress of green LED die development using $\mathrm{Ga}_{1-\mathrm{x}} \mathrm{In}_{\mathrm{x}} \mathrm{N} / \mathrm{GaN}$ quantum wells optimized for high lateral homogeneity as established by low surface roughness in AFM. Under further optimization of the growth processes we anticipate that efficient commercial viable LEDs covering the entire green spectral range will become possible.

\section{References}

[1] I. Akasaki, Mater. Res. Soc. Symp. Proc. 482, 1 (1998).

[2] S. Nakamura and G. Fasol, The Blue Laser Diode (Springer, Berlin, 1997).

[3] I. Akasaki and H. Amano, in: High Brightness Light Emitting Diodes, edited by G.B. Stringfellow and M.G. Craford (Semiconductors and Semimetals, Vol. 48, Academic Press, London, 1997), p. 357.

[4] T. Mukai, M. Yamada, and S. Nakamura, Jpn. J. Appl. Phys. 38, 3976 (1999).

[5] C. Wetzel, T. Salagaj, T. Detchprohm, P. Li, and J.S. Nelson, Appl. Phys. Lett. 85, 866 (2004).

[6] X.H. Wu, C.R. Elsass, A. Abare, M. Mack, S. Keller, P.M. Petroff, S.P. DenBaars, J.S. Speck, and S.J. Rosner, Appl. Phys. Lett. 72, 692 (1998). 